\title{
Proof of the fundamental BCJ relations for QCD amplitudes
}

\section{Leonardo de la Cruz, Alexander Kniss and Stefan Weinzierl}

PRISMA Cluster of Excellence, Institut für Physik, Johannes Gutenberg-Universität Mainz, Staudingerweg 7, Mainz, D-55099 Germany

E-mail: ledelacr@uni-mainz.de, akniss@students.uni-mainz.de, weinzierl@uni-mainz.de

ABSTRACT: The fundamental BCJ-relation is a linear relation between primitive tree amplitudes with different cyclic orderings. The cyclic orderings differ by the insertion place of one gluon. The coefficients of the fundamental BCJ-relation are linear in the Lorentz invariants $2 p_{i} p_{j}$. The BCJ-relations are well established for pure gluonic amplitudes as well as for amplitudes in $\mathcal{N}=4$ super-Yang-Mills theory. Recently, it has been conjectured that the BCJ-relations hold also for QCD amplitudes. In this paper we give a proof of this conjecture. The proof is valid for massless and massive quarks.

Keywords: Scattering Amplitudes, QCD

ARXiv EPrint: 1508.01432 


\section{Contents}

1 Introduction 2

2 Overview 3

3 Momentum deformation 5

3.1 Spinor definitions 5

3.2 Decomposition of massive four-vectors into light-like four-vectors $\quad 7$

$\begin{array}{lll}3.3 & \text { On the choice of the reference spinors } & 7\end{array}$

3.4 BCFW-shifts 8

3.4.1 The helicity configuration $1^{+}, 2_{g}^{+}, n^{-} \quad 9$

3.4.2 The helicity configuration $1^{+}, 2_{g}^{-}, n^{-} \quad 9$

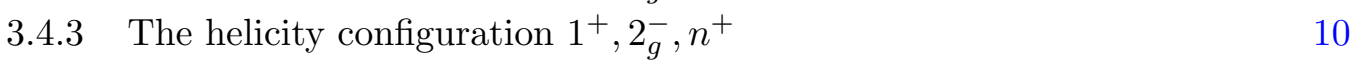

3.4.4 The helicity configuration $1^{+}, 2_{g}^{+}, n^{+} \quad 11$

3.4.5 The remaining helicity configurations 11

4 Large- $z$ behaviour $\quad 11$

4.1 Three gluons 12

$\begin{array}{ll}4.2 & \text { Two gluons and one quark/anti-quark } \\ \end{array}$

4.3 One gluon and two quarks/anti-quarks, not belonging to the same fermion line 12

4.4 One gluon and a quark-anti-quark-pair belonging to the same fermion line 13

5 The proof by induction $\quad 14$

$\begin{array}{ll}5.1 & \text { Induction start: the case } n=3\end{array}$

$\begin{array}{lll}5.2 & \text { The induction step } & 15\end{array}$

$\begin{array}{llr}6 & \text { Conclusions } & 18\end{array}$

$\begin{array}{ll}\text { A Reference spinors for massive particles } & 18\end{array}$

$\begin{array}{lll}\text { A.1 The helicity configuration } 1^{+}, 2_{g}^{+}, n^{-} & 18\end{array}$

$\begin{array}{ll}\text { A.2 The helicity configuration } 1^{+}, 2_{g}^{-}, n^{-} & 18\end{array}$

B The large $z$-behaviour in the eikonal approximation $\quad 19$

$\begin{array}{ll}\text { C Cyclic-ordered Feynman rules } & 21\end{array}$ 


\section{Introduction}

Amplitudes in QCD are often computed by a decomposition into a sum of smaller pieces, called primitive amplitudes $[1,2]$. The primitive amplitudes are gauge invariant, colourstripped and have a fixed ordering of the external legs. Non-trivial relations between different primitive tree amplitudes are a fascinating topic and have important applications. For pure gluonic primitive tree amplitudes these relations are by now well-studied. Invariance under cyclic permutations is trivial. The first non-trivial relations are the Kleiss-Kuijf relations [3], which follow from the anti-symmetry of the colour-stripped vertices. More interesting are the Bern-Carrasco-Johansson relations (BCJ-relations) [4]. The full set of the BCJ-relations follows from the so-called fundamental BCJ-relations [5]. The fundamental BCJ-relation is a linear relation between primitive tree amplitudes with different cyclic orderings. The cyclic orderings differ by the insertion place of one gluon. In the fundamental BCJ-relation the coefficients of the relation are linear in the Lorentz invariants $2 p_{i} p_{j}$. The BCJ-relations are known to hold for pure gluonic tree amplitudes and for tree amplitudes in $\mathcal{N}=4$ super-Yang-Mills theory. The BCJ relations have been proven first with methods from string theory $[6,7]$ and later within quantum field theory with the help of on-shell recursion relations $[5,8,9]$. On-shell recursion relations require a certain fall-off behaviour for large momentum deformations. For amplitudes in $\mathcal{N}=4 \mathrm{SYM}$ it is essential that not only the (bosonic) momentum components but also the (fermionic) Grassmann components are shifted. The required fall-off behaviour has been established for pure gluonic tree amplitudes and amplitudes in $\mathcal{N}=4$ SYM in [10-12]. Furthermore BCJ relations have been derived for a pair of massive scalars and an arbitrary number of gluons [13].

It is natural to consider primitive tree amplitudes in QCD as well, involving in addition to gluons massless and/or massive quarks $[14,15]$. The fundamental BCJ-relation singles out three particles, which we will label 1, 2 and $n$. In the fundamental BCJ-relation the positions of particles 1 and $n$ are fixed, as there are positions of the remaining particles 3 to $(n-1)$. In the cyclic order particles 1 and $n$ are adjacent. In the fundamental BCJ-relation particle 2 is inserted in all possible places in the cyclic order between 1 and $n$, but not between $n$ and 1. Recently, Johansson and Ochirov conjectured [14] that the fundamental BCJ-relations hold for primitive tree amplitudes in full QCD as well, provided particle 2 is a gluon. In this paper we prove this conjecture. The major part of our arguments is not specific to four space-time dimensions. Only in the explicit definitions of the momentum shifts we use spinor representations corresponding to four space-time dimensions. With a suitable generalisation of these momentum shifts our proof will carry over to $D$ space-time dimensions.

This paper is organised as follows: in section 2 we introduce the conjecture, which we are going to prove and give an outline of the proof. Section 3 carefully defines momentum deformations through three-particle shifts. This is a necessary technical preparation for our proof. In section 4 we discuss the large $z$-behaviour of the deformed fundamental BCJ-relation under the three-particle shifts and show that there is no contribution from infinity in BCFW-recursion relations. In section 5 we prove the fundamental BCJ-relation 
by induction. Our conclusions are given in section 6 . In an appendix we collected some technical details on certain three-particle shifts with massive quarks (appendix A) and a proof on the large $z$-behaviour in the eikonal approximation (appendix B). For the convenience of the reader we also included the cyclic-ordered Feynman rules relevant to primitive amplitudes in appendix $\mathrm{C}$.

\section{Overview}

Tree amplitudes in QCD with any number of external quarks can be decomposed systematically into gauge-invariant primitive amplitudes with a fixed cyclic ordering $[2,16,17]$. Let us consider a tree-level primitive QCD amplitude with $n$ external particles, out of which $n_{q}$ particles are quarks, $n_{q}$ particles are anti-quarks and $n_{g}$ particles are gluons. We have the obvious relation

$$
n=n_{g}+2 n_{q}
$$

Without loss of generality we may assume that all quarks have different flavours. The quarks may be massless or massive. In this paper we consider amplitudes with at least one gluon $\left(n_{g}>0\right)$. This excludes the case, where all external particles are either quarks or anti-quarks $\left(n_{g}=0\right.$ and hence $\left.n=2 n_{q}\right)$. We are mainly interested in the case, where there is in addition to gluons at least one quark-anti-quark pair $\left(n_{q}>0\right)$. The pure gluonic case $\left(n_{q}=0\right)$ is not excluded, but already well studied. The tree-level primitive QCD amplitudes are cyclic-ordered. We denote such an amplitude with the cyclic-order $(1,2, \ldots, n)$ by

$$
A_{n}(1,2, \ldots, n)
$$

The amplitudes satisfy the Kleiss-Kuijf relations [3]. In order to state the Kleiss-Kuijf relation let

$$
\vec{\alpha}=\left(\alpha_{1}, \ldots, \alpha_{j}\right), \quad \vec{\beta}=\left(\beta_{1}, \ldots, \beta_{n-2-j}\right)
$$

and $\vec{\beta}^{T}=\left(\beta_{n-2-j}, \ldots, \beta_{1}\right)$. The Kleiss-Kuijf relations read

$$
A_{n}(1, \vec{\beta}, 2, \vec{\alpha})=(-1)^{n-2-j} \sum_{\sigma \in \vec{\alpha} \amalg \vec{\beta}^{T}} A_{n}\left(1,2, \sigma_{1}, \ldots, \sigma_{n-2}\right) .
$$

Here, $\vec{\alpha} \amalg \vec{\beta}^{T}$ denotes the set of all shuffles of $\vec{\alpha}$ with $\vec{\beta}^{T}$, i.e. the set of all permutations of the elements of $\vec{\alpha}$ and $\vec{\beta}^{T}$, which preserve the relative order of the elements of $\vec{\alpha}$ and of the elements of $\vec{\beta}^{T}$. A special case of the Kleiss-Kuijf relations is the situation, where the set $\beta$ contains only one element. In this case the Kleiss-Kuijf relation reduces to the $\mathrm{U}(1)$-decoupling identity

$$
\sum_{\sigma \in \mathbb{Z}_{n-1}} A_{n}\left(\sigma_{1}, \sigma_{2}, \ldots, \sigma_{n-1}, n\right)=0
$$


where the sum is over the cyclic permutations of the first $(n-1)$ arguments. The KleissKuijf relations in eq. (2.4) allow us to fix two legs at specified positions. We will use this freedom to fix a particle at position 1 and a second particle at position $n$. Since we also assume that there is at least one gluon, let us label this gluon by $2_{g}$. In [14] Johansson and Ochirov conjecture that

$$
\sum_{i=2}^{n-1}\left(\sum_{j=i+1}^{n} 2 p_{2} p_{j}\right) A_{n}\left(1,3, \ldots, i, 2_{g}, i+1, \ldots, n-1, n\right)=0 .
$$

In this paper we prove this conjecture. An equivalent formulation of eq. (2.6) is

$$
\sum_{i=2}^{n-1}\left(\sum_{j=1}^{i} 2 p_{2} p_{j}\right) A_{n}\left(1,3, \ldots, i, 2_{g}, i+1, \ldots, n-1, n\right)=0,
$$

which follows from eq. (2.6) by momentum conservation. Eq. (2.6) is the fundamental BCJrelation $[4,5]$ for tree-level primitive QCD amplitudes. It is well known that eq. (2.6) holds in the pure gluonic case. It is also know that eq. (2.6) holds for amplitudes with one quarkanti-quark pair $\left(n_{q}=1\right)$ in massless QCD. This follows from the fact that these amplitudes are identical to the corresponding amplitudes in $\mathcal{N}=4$ super-Yang-Mills theory, and the fact that the BCJ-relations hold for the latter $[8,11] .{ }^{1}$ The aim of this paper is to show that eq. (2.6) holds more generally in (massless or massive) QCD.

In order to prove the fundamental BCJ-relation we will make use of on-shell recursion relations (or Britto-Cachazo-Feng-Witten recursion relations, BCFW-recursion relations for short) [21]. Within on-shell recursion relations some distinguished external momenta are deformed, such that momentum conservation and the on-shell conditions are respected. Let us consider a deformation of the momenta $p_{1}, p_{2}$ and $p_{n}$ depending on a variable $z$. This is called a three-particle BCFW-shift [22]. Since for our problem there are three distinguished particles $1,2_{g}$ and $n$, a three-particle shift is more natural as compared to a more conventional two-particle shift. It will turn out that a three-particle shift will simplify the proof. We denote the deformed momenta by

$$
\hat{p}_{1}(z), \quad \hat{p}_{2}(z), \quad \hat{p}_{n}(z)
$$

We further require

$$
\hat{p}_{1}(0)=p_{1}, \quad \hat{p}_{2}(0)=p_{2}, \quad \hat{p}_{n}(0)=p_{n} .
$$

For $j \neq 1,2, n$ we simply set $\hat{p}_{j}(z)=p_{j}$. We introduce the quantity

$$
I_{n}(z)=\sum_{i=2}^{n-1}\left(\sum_{j=i+1}^{n} 2 \hat{p}_{2} \hat{p}_{j}\right) A_{n}\left(\hat{1}, 3, \ldots, i, \hat{2}_{g}, i+1, \ldots, n-1, \hat{n}\right) .
$$

\footnotetext{
${ }^{1}$ It is worth noting that all tree amplitudes in massless QCD can be obtained from tree amplitudes in $\mathcal{N}=4$ super-Yang-Mills theory [18-20].
} 
For $z=0$ the expression $I_{n}(z)$ reduces to the left-hand-side of eq. (2.6). $I_{n}(z)$ is clearly a rational function of $z$. We have to show

$$
I_{n}(0)=0,
$$

or equivalently

$$
\frac{1}{2 \pi i} \oint_{z=0} \frac{d z}{z} I_{n}(z)=0 .
$$

We do this in two steps: we first show that $I_{n}(z)$ has no pole at $z=\infty$, or equivalently, that $I_{n}(z)$ falls off for large $z$ at least with $1 / z$. In the second step we use induction and $\mathrm{BCFW}$-recursion in order to prove eq. (2.11).

\section{Momentum deformation}

In this section we review the three-particle BCFW-shift for the external particles $1,2_{g}$ and $n$. The types of particles 1 and $n$ may either be a quark, an anti-quark or a gluon. However, particle $2_{g}$ is required to be a gluon. Quarks and anti-quarks may be massive or massless. Therefore we will treat the general case with arbitrary masses for particles 1 and $n$. The case of massless quarks is included as the special case $m_{1}=m_{n}=0$. This section may appear at a first reading a little bit technical, but actually it will pay off: we define the momentum deformations in such a way, that we obtain the same large $z$ behaviour independently of the helicity configuration and independently of the masses of the particles. Most of the technical details are related to massive quarks and it might be advantageous at a first reading to pay attention to the massless case only. In the massless case the formulae simplify considerably.

\subsection{Spinor definitions}

For the definition of massive spinors we follow [23]. Let us consider two independent Weyl spinors $|q+\rangle$ and $\langle q+|$. These two Weyl spinors define a light-like four-vector

$$
q^{\mu}=\frac{1}{2}\left\langle q+\left|\gamma^{\mu}\right| q+\right\rangle
$$

This four-vector can be used to associate to any not necessarily light-like four-vector $p$ a light-like four-vector $p^{b}$ :

$$
p^{b}=p-\frac{p^{2}}{2 p \cdot q} q .
$$

The four-vector $p^{b}$ satisfies $\left(p^{b}\right)^{2}=0$. Note that $p^{b}$ depends implicitly on $|q+\rangle$ and $\langle q+|$. The two Weyl spinors $|q+\rangle$ and $\langle q+|$ are used as reference spinors in the definition of the polarisations of the external particles. For quarks with momentum $p$ we take the spinors $u$ and $\bar{u}$ as

$$
\begin{array}{ll}
u(-)=\frac{1}{\left\langle p^{b}+\mid q-\right\rangle}(\not p+m)|q-\rangle, & \bar{u}(+)=\frac{1}{\left\langle q-\mid p^{b}+\right\rangle}\langle q-|(\not p+m), \\
u(+)=\frac{1}{\left\langle p^{b}-\mid q+\right\rangle}(\not p+m)|q+\rangle, & \bar{u}(-)=\frac{1}{\left\langle q+\mid p^{b}-\right\rangle}\langle q+|(\not p+m) .
\end{array}
$$


The spinors $v$ and $\bar{v}$ are given by

$$
\begin{array}{ll}
v(-)=\frac{1}{\left\langle p^{b}+\mid q-\right\rangle}(\not p-m)|q-\rangle, & \bar{v}(+)=\frac{1}{\left\langle q-\mid p^{b}+\right\rangle}\langle q-|(\not p-m), \\
v(+)=\frac{1}{\left\langle p^{b}-\mid q+\right\rangle}(\not p-m)|q+\rangle, & \bar{v}(-)=\frac{1}{\left\langle q+\mid p^{b}-\right\rangle}\langle q+|(\not p-m) .
\end{array}
$$

We label the helicities as if all particles were outgoing. As a consequence, the spinors $u(\lambda)$ and $\bar{v}(\lambda)$, which correspond to particles with incoming momentum, have a reversed helicity assignment. In the massless limit the definition reduces to

$$
\begin{array}{ll}
u(-)=v(-)=|p+\rangle, & \bar{u}(+)=\bar{v}(+)=\langle p+|, \\
u(+)=v(+)=|p-\rangle, & \bar{u}(-)=\bar{v}(-)=\langle p-|,
\end{array}
$$

and the spinors are independent of the reference spinors $|q+\rangle$ and $\langle q+|$.

For massive fermions the reference spinors are related to the quantisation axis of the spin for this fermion, and the individual amplitudes with label + or - will therefore depend on the reference spinors $|q+\rangle$ and $\langle q+|$. It should be noted that the spinors for the massive fermions depend both on $|q+\rangle$ and $\langle q+|$ : for the spinors with helicity " + " there is an explicit dependence on $|q+\rangle$, while the dependence on $\langle q+|$ enters implicitly through $p^{b}$. In a similar way we find that the spinors with helicity "-" have an explicit dependence on $\langle q+|$, while the dependence on $|q+\rangle$ enters implicitly through $p^{b}$.

It is easy to relate helicity amplitudes of massive quarks corresponding to one choice of reference spinors to another set of reference spinors. If $|\tilde{q}+\rangle$ and $\langle\tilde{q}+|$ is a second pair of reference spinors we have the following transformation law

$$
\left(\begin{array}{l}
\bar{u}(+, \tilde{q}) \\
\bar{u}(-, \tilde{q})
\end{array}\right)=\left(\begin{array}{ll}
c_{11} & c_{12} \\
c_{21} & c_{22}
\end{array}\right)\left(\begin{array}{l}
\bar{u}(+, q) \\
\bar{u}(-, q)
\end{array}\right),
$$

where

$$
c_{11}=\frac{\langle\tilde{q}-|p| q-\rangle}{\left\langle\tilde{q} \tilde{p}^{b}\right\rangle\left[p^{b} q\right]}, \quad c_{12}=\frac{m\langle\tilde{q} q\rangle}{\left\langle\tilde{q} \tilde{p}^{b}\right\rangle\left\langle p^{b} q\right\rangle}, \quad c_{21}=\frac{m[\tilde{q} q]}{\left[\tilde{q} \tilde{p}^{b}\right]\left[p^{b} q\right]}, \quad c_{22}=\frac{\langle\tilde{q}+|p| q+\rangle}{\left[\tilde{q} \tilde{p}^{b}\right]\left\langle p^{b} q\right\rangle} .
$$

Here, $\tilde{p}^{b}$ denotes the projection onto a light-like four-vector with respect to the reference vector $\frac{1}{2}\left\langle\tilde{q}+\left|\gamma^{\mu}\right| \tilde{q}+\right\rangle$. Similar, we have for an amplitude with an incoming massive quark

$$
\left(\begin{array}{l}
u(+, \tilde{q}) \\
u(-, \tilde{q})
\end{array}\right)=\left(\begin{array}{cc}
c_{11} & -c_{12} \\
-c_{21} & c_{22}
\end{array}\right)\left(\begin{array}{l}
u(+, q) \\
u(-, q)
\end{array}\right) .
$$

Similar formulae exist for the spinors $v$ and $\bar{v}$ and can be obtained by the substitution $u \rightarrow v, \bar{u} \rightarrow \bar{v}$ and $m \rightarrow-m$.

For the polarisation vectors of a gluon with momentum $p$ we take

$$
\varepsilon_{\mu}^{+}=\frac{\left\langle p+\left|\gamma_{\mu}\right| q+\right\rangle}{\sqrt{2}\langle q-\mid p+\rangle}, \quad \varepsilon_{\mu}^{-}=\frac{\left\langle q+\left|\gamma_{\mu}\right| p+\right\rangle}{\sqrt{2}\langle p+\mid q-\rangle} .
$$

The dependence on the reference spinors $|q+\rangle$ and $\langle q+|$, which enters through the gluon polarisation vectors will drop out in gauge invariant quantities. 


\subsection{Decomposition of massive four-vectors into light-like four-vectors}

The external momenta of particles 1 and $n$ may be massive or massless. In the case where they are massive (either one of them or both) we would like to write them as a linear combination of two light-like four-vectors $l_{1}$ and $l_{n}$. The two light-like four-vectors $l_{1}$ and $l_{n}$ are constructed as follows $[24,25]$ : if $p_{1}$ and $p_{n}$ are massless, $l_{1}$ and $l_{n}$ are given by

$$
l_{1}=p_{1}, \quad l_{n}=p_{n} .
$$

If $p_{1}$ is massless, but $p_{n}$ is massive one has

$$
l_{1}=p_{1}, \quad l_{n}=-\alpha_{1} p_{1}+p_{n}, \quad \alpha_{1}=\frac{p_{n}^{2}}{2 p_{1} p_{n}} .
$$

The inverse formula is given by

$$
p_{1}=l_{1}, \quad p_{n}=\alpha_{1} l_{1}+l_{n} .
$$

If both $p_{1}$ and $p_{n}$ are massive, one has

$$
l_{1}=\frac{1}{1-\alpha_{1} \alpha_{n}}\left(p_{1}-\alpha_{n} p_{n}\right), \quad l_{n}=\frac{1}{1-\alpha_{1} \alpha_{n}}\left(-\alpha_{1} p_{1}+p_{n}\right) .
$$

$\alpha_{1}$ and $\alpha_{n}$ are given by

$$
\alpha_{1}=\frac{2 p_{1} p_{n}-\operatorname{sign}\left(2 p_{1} p_{n}\right) \sqrt{\Delta}}{2 p_{1}^{2}}, \quad \alpha_{n}=\frac{2 p_{1} p_{n}-\operatorname{sign}\left(2 p_{1} p_{n}\right) \sqrt{\Delta}}{2 p_{n}^{2}} .
$$

Here,

$$
\Delta=\left(2 p_{1} p_{n}\right)^{2}-4 p_{1}^{2} p_{n}^{2}
$$

The signs are chosen in such away that the massless limit $p_{1}^{2} \rightarrow 0$ (or $p_{n}^{2} \rightarrow 0$ ) is approached smoothly. The inverse formulae are given by

$$
p_{1}=l_{1}+\alpha_{n} l_{n}, \quad p_{n}=\alpha_{1} l_{1}+l_{n} .
$$

The light-like four-vectors $l_{1}$ and $l_{n}$ define massless spinors $\left|l_{1}+\right\rangle,\left\langle l_{1}+|,| l_{n}+\right\rangle$ and $\left\langle l_{n}+\right|$.

\subsection{On the choice of the reference spinors}

Particles 1 and $n$ may be massive quarks or anti-quarks and we have to make a choice for the reference spinors. In the massless case, the amplitude will be independent of the choice of the reference spinors and this section is of no further relevance. However, if particle 1 (or particle $n$ ) is massive, the choice of the reference spinors will define the spin quantisation axis and the amplitude will depend on this choice. It is always possible to convert to a different basis with the help of eqs. (3.6)-(3.8).

In section 3.2 we have constructed the spinors $\left|l_{1}+\right\rangle,\left|l_{n}+\right\rangle,\left\langle l_{1}+\right|$ and $\left\langle l_{n}+\right|$. For generic momenta $p_{1}$ and $p_{n}$, the ket-spinors $\left|l_{1}+\right\rangle$ and $\left|l_{n}+\right\rangle$ span the two-dimensional space of holomorphic spinors (or ket-plus-spinors). Similarly, the bra-spinors $\left\langle l_{1}+\right|$ and 
$\left\langle l_{n}+\right|$ span the space of anti-holomorphic spinors (or bra-plus-spinors). For particle 1 we parametrise the reference spinors $\left|q_{1}+\right\rangle$ and $\left\langle q_{1}+\right|$ as

$$
\left|q_{1}+\right\rangle=\left|l_{n}+\right\rangle+\lambda_{1}\left|l_{1}+\right\rangle, \quad\left\langle q_{1}+\right|=\left\langle l_{n}+\right|+\lambda_{1}\left\langle l_{1}+\right|,
$$

with one parameter $\lambda_{1}$. For particle $n$ we parametrise the reference spinors $\left|q_{n}+\right\rangle$ and $\left\langle q_{n}+\right|$ as

$$
\left|q_{n}+\right\rangle=\left|l_{1}+\right\rangle+\lambda_{n}\left|l_{n}+\right\rangle, \quad\left\langle q_{n}+\right|=\left\langle l_{1}+\right|+\lambda_{n}\left\langle l_{n}+\right|,
$$

with one further parameter $\lambda_{n}$. Working out $\left|p_{1}^{b}+\right\rangle,\left|p_{n}^{b}+\right\rangle,\left\langle p_{1}^{b}+\right|$ and $\left\langle p_{n}^{b}+\right|$ one finds

$$
\begin{array}{ll}
\left|p_{1}^{b}+\right\rangle=c_{1}\left(\left|l_{1}+\right\rangle-\alpha_{n} \lambda_{1}\left|l_{n}+\right\rangle\right), & \left\langle p_{1}^{b}+\right|=c_{1}\left(\left\langle l_{1}+\right|-\alpha_{n} \lambda_{1}\left\langle l_{n}+\right|\right), \\
\left|p_{n}^{b}+\right\rangle=c_{n}\left(\left|l_{n}+\right\rangle-\alpha_{1} \lambda_{n}\left|l_{1}+\right\rangle\right), & \left\langle p_{n}^{b}+\right|=c_{n}\left(\left\langle l_{n}+\right|-\alpha_{1} \lambda_{n}\left\langle l_{1}+\right|\right),
\end{array}
$$

with

$$
c_{1}=\frac{1}{\sqrt{1+\alpha_{n} \lambda_{1}^{2}}}, \quad c_{n}=\frac{1}{\sqrt{1+\alpha_{1} \lambda_{n}^{2}}} .
$$

The variables $\alpha_{1}$ and $\alpha_{n}$ have been defined in eq. (3.14). We will use the freedom to choose $\lambda_{1}$ and $\lambda_{n}$ to compensate a restriction on the possible BCFW-shifts for massive particles. The shifted spinors of the massive particles have to satisfy the Dirac equation, as well as orthogonality and completeness relations. This restricts the $z$-dependent part to be proportional to the corresponding reference spinors [23]. This means if we shift a massive spinor

$$
\hat{u}(-)=u(-)-z|\eta+\rangle,
$$

the spinor $|\eta+\rangle$ has to be proportional to $|q+\rangle$ :

$$
|\eta+\rangle=\kappa|q+\rangle .
$$

Similarly, if we shift

$$
\hat{\bar{u}}(+)=\bar{u}(+)+z\langle\eta+|
$$

we have to require that

$$
\langle\eta+|=\kappa\langle q+| .
$$

\subsection{BCFW-shifts}

We now provide the explicit formulae for the three-particle shifts. We have to consider all possible helicity configurations for the three particles $1,2_{g}$ and $n$. In all cases the deformations are defined in such a way, that the external polarisations spinors and vectors give the best possible large $z$-behaviour. This is a $z^{-3}$-behaviour if the three particles 1 , $2_{g}$ and $n$ are all gluons, a $z^{-2}$-behaviour if one of them is a quark or an anti-quark and a $z^{-1}$-behaviour if two of them are quarks or anti-quarks. As particle $2_{g}$ is required to be a gluon, the case where all three particles are quarks or anti-quarks is not possible. The large $z$-behaviour of the external polarisations is summarised in table 1 . 


\begin{tabular}{|l|l|}
\hline particles $1,2_{g}$ and $n$ & large $z$-behaviour \\
\hline 3 gluons & $z^{-3}$ \\
2 gluons, 1 quark/anti-quark & $z^{-2}$ \\
1 gluon, 2 quarks/anti-quarks & $z^{-1}$ \\
\hline
\end{tabular}

Table 1. The large $z$-behaviour of the external polarisations under the three-particle shifts.

\subsubsection{The helicity configuration $1^{+}, 2_{g}^{+}, n^{-}$}

For the helicity configuration $1^{+}, 2_{g}^{+}, n^{-}$we shift $u_{1}(-), u_{2}(-)$ and $\bar{u}_{n}(+)$, while $\bar{u}_{1}(+)$, $\bar{u}_{2}(+)$ and $u_{n}(-)$ remain unchanged:

$$
\begin{aligned}
& \hat{u}_{1}(-)=u_{1}(-)-z y_{1}\left|p_{n}^{b}+\right\rangle, \quad \hat{\bar{u}}_{n}(+)=\bar{u}_{n}(+)+z y_{1}\left\langle p_{1}^{b}+\right|+z y_{2}\left\langle p_{2}+\right|, \\
& \hat{u}_{2}(-)=u_{2}(-)-z y_{2}\left|p_{n}^{b}+\right\rangle,
\end{aligned}
$$

where $y_{1}$ and $y_{2}$ are two non-zero constants. For massive particles we have to require, that the shift is proportional to the corresponding reference spinors. Therefore we have to require that the system of equations

$$
\kappa_{1}\left|q_{1}+\right\rangle=y_{1}\left|p_{n}^{b}+\right\rangle, \quad \kappa_{n}\left\langle q_{n}+\right|=y_{1}\left\langle p_{1}^{b}+\right|+y_{2}\left\langle p_{2}+\right|,
$$

has a solution for some non-zero constants $\kappa_{1}$ and $\kappa_{n}$. In appendix A.1 we show that this is the case. The spinors $\hat{u}_{1}(-)$ and $\hat{\bar{u}}_{1}(+)$ correspond to an on-shell particle with mass $m_{1}$ and four-momentum

$$
\hat{p}_{1}^{\mu}=p_{1}^{\mu}-\frac{1}{2} z y_{1}\left\langle p_{1}^{b}+\left|\gamma^{\mu}\right| p_{n}^{b}+\right\rangle .
$$

The spinors $\hat{u}_{2}(-)$ and $\hat{\bar{u}}_{2}(+)$ correspond to an on-shell particle with zero mass and fourmomentum

$$
\hat{p}_{2}^{\mu}=p_{2}^{\mu}-\frac{1}{2} z y_{2}\left\langle p_{2}+\left|\gamma^{\mu}\right| p_{n}^{b}+\right\rangle .
$$

The spinors $\hat{u}_{n}(-)$ and $\hat{\bar{u}}_{n}(+)$ correspond to an on-shell particle with mass $m_{n}$ and fourmomentum

$$
\hat{p}_{n}^{\mu}=p_{n}^{\mu}+\frac{1}{2} z y_{1}\left\langle p_{1}^{b}+\left|\gamma^{\mu}\right| p_{n}^{b}+\right\rangle+\frac{1}{2} z y_{2}\left\langle p_{2}+\left|\gamma^{\mu}\right| p_{n}^{b}+\right\rangle .
$$

\subsubsection{The helicity configuration $1^{+}, 2_{g}^{-}, n^{-}$}

For the helicity configuration $1^{+}, 2_{g}^{-}, n^{-}$we shift $u_{1}(-), \bar{u}_{2}(+)$ and $\bar{u}_{n}(+)$, while $\bar{u}_{1}(+)$, $u_{2}(-)$ and $u_{n}(-)$ remain unchanged:

$$
\begin{array}{ll}
\hat{u}_{1}(-)=u_{1}(-)-z y_{2}\left|p_{2}+\right\rangle-z y_{n}\left|p_{n}^{b}+\right\rangle, & \hat{\bar{u}}_{2}(+)=\bar{u}_{2}(+)+z y_{2}\left\langle p_{1}^{b}+\right|, \\
& \hat{\bar{u}}_{n}(+)=\bar{u}_{n}(+)+z y_{n}\left\langle p_{1}^{b}+\right|,
\end{array}
$$


where $y_{2}$ and $y_{n}$ are two non-zero constants. For massive particles we have to require that the system of equations

$$
\kappa_{1}\left|q_{1}+\right\rangle=y_{2}\left|p_{2}+\right\rangle+y_{n}\left|p_{n}^{b}+\right\rangle, \quad \kappa_{n}\left\langle q_{n}+\right|=y_{n}\left\langle p_{1}^{b}+\right|,
$$

has a solution for some non-zero constants $\kappa_{1}$ and $\kappa_{n}$. In appendix A.2 we show that this is the case. The spinors $\hat{u}_{1}(-)$ and $\hat{\bar{u}}_{1}(+)$ correspond to an on-shell particle with mass $m_{1}$ and four-momentum

$$
\hat{p}_{1}^{\mu}=p_{1}^{\mu}-\frac{1}{2} z y_{2}\left\langle p_{1}^{b}+\left|\gamma^{\mu}\right| p_{2}+\right\rangle-\frac{1}{2} z y_{n}\left\langle p_{1}^{b}+\left|\gamma^{\mu}\right| p_{n}^{b}+\right\rangle .
$$

The spinors $\hat{u}_{2}(-)$ and $\hat{\bar{u}}_{2}(+)$ correspond to an on-shell particle with zero mass and fourmomentum

$$
\hat{p}_{2}^{\mu}=p_{2}^{\mu}+\frac{1}{2} z y_{2}\left\langle p_{1}^{b}+\left|\gamma^{\mu}\right| p_{2}+\right\rangle .
$$

The spinors $\hat{u}_{n}(-)$ and $\hat{\bar{u}}_{n}(+)$ correspond to an on-shell particle with mass $m_{n}$ and fourmomentum

$$
\hat{p}_{n}^{\mu}=p_{n}^{\mu}+\frac{1}{2} z y_{n}\left\langle p_{1}^{b}+\left|\gamma^{\mu}\right| p_{n}^{b}+\right\rangle .
$$

\subsubsection{The helicity configuration $1^{+}, 2_{g}^{-}, n^{+}$}

For the helicity configuration $1^{+}, 2_{g}^{-}, n^{+}$we shift $u_{1}(-), \bar{u}_{2}(+)$ and $u_{n}(-)$, while $\bar{u}_{1}(+)$, $u_{2}(-)$ and $\bar{u}_{n}(+)$ remain unchanged:

$$
\begin{aligned}
& \hat{u}_{1}(-)=u_{1}(-)-z y_{1}\left|p_{2}+\right\rangle, \quad \hat{\bar{u}}_{2}(+)=\bar{u}_{2}(+)+z y_{1}\left\langle p_{1}^{b}+\right|+z y_{n}\left\langle p_{n}^{b}+\right|, \\
& \hat{u}_{n}(-)=u_{n}(-)-z y_{n}\left|p_{2}+\right\rangle,
\end{aligned}
$$

where $y_{1}$ and $y_{n}$ are two non-zero constants. For massive particles we choose

$$
\left|q_{1}+\right\rangle=\left|q_{n}+\right\rangle=\left|p_{2}+\right\rangle, \quad\left\langle q_{1}+\right|=\left\langle q_{n}+\right|=\left\langle p_{2}+\right|
$$

as reference spinors. The spinors $\hat{u}_{1}(-)$ and $\hat{\bar{u}}_{1}(+)$ correspond to an on-shell particle with mass $m_{1}$ and four-momentum

$$
\hat{p}_{1}^{\mu}=p_{1}^{\mu}-\frac{1}{2} z y_{1}\left\langle p_{1}^{b}+\left|\gamma^{\mu}\right| p_{2}+\right\rangle .
$$

The spinors $\hat{u}_{2}(-)$ and $\hat{\bar{u}}_{2}(+)$ correspond to an on-shell particle with zero mass and fourmomentum

$$
\hat{p}_{2}^{\mu}=p_{2}^{\mu}+\frac{1}{2} z y_{1}\left\langle p_{1}^{b}+\left|\gamma^{\mu}\right| p_{2}+\right\rangle+\frac{1}{2} z y_{n}\left\langle p_{n}^{b}+\left|\gamma^{\mu}\right| p_{2}+\right\rangle .
$$

The spinors $\hat{u}_{n}(-)$ and $\hat{\bar{u}}_{n}(+)$ correspond to an on-shell particle with mass $m_{n}$ and fourmomentum

$$
\hat{p}_{n}^{\mu}=p_{n}^{\mu}-\frac{1}{2} z y_{n}\left\langle p_{n}^{b}+\left|\gamma^{\mu}\right| p_{2}+\right\rangle
$$




\subsubsection{The helicity configuration $1^{+}, 2_{g}^{+}, n^{+}$}

For the helicity configuration $1^{+}, 2_{g}^{+}, n^{+}$we shift $u_{1}(-), u_{2}(-)$ and $u_{n}(-)$, while $\bar{u}_{1}(+)$, $\bar{u}_{2}(+)$ and $\bar{u}_{n}(+)$ remain unchanged:

$$
\begin{aligned}
& \hat{u}_{1}(-)=u_{1}(-)-z\left[p_{2} p_{n}^{b}\right]|\eta+\rangle, \\
& \hat{u}_{2}(-)=u_{2}(-)-z\left[p_{n}^{b} p_{1}^{b}\right]|\eta+\rangle, \\
& \hat{u}_{n}(-)=u_{n}(-)-z\left[p_{1}^{b} p_{2}\right]|\eta+\rangle .
\end{aligned}
$$

Here, $|\eta+\rangle$ is an arbitrary spinor. For massive particles we choose

$$
\left|q_{1}+\right\rangle=\left|q_{n}+\right\rangle=|\eta+\rangle, \quad\left\langle q_{1}+\right|=\left\langle q_{n}+\right|=\langle\eta+|
$$

as reference spinors. The spinors $\hat{u}_{1}(-)$ and $\hat{\bar{u}}_{1}(+)$ correspond to an on-shell particle with mass $m_{1}$ and four-momentum

$$
\hat{p}_{1}^{\mu}=p_{1}^{\mu}-\frac{1}{2} z\left[p_{2} p_{n}^{b}\right]\left\langle p_{1}^{b}+\left|\gamma^{\mu}\right| \eta+\right\rangle .
$$

The spinors $\hat{u}_{2}(-)$ and $\hat{\bar{u}}_{2}(+)$ correspond to an on-shell particle with zero mass and fourmomentum

$$
\hat{p}_{2}^{\mu}=p_{2}^{\mu}-\frac{1}{2} z\left[p_{n}^{b} p_{1}^{b}\right]\left\langle p_{2}+\left|\gamma^{\mu}\right| \eta+\right\rangle .
$$

The spinors $\hat{u}_{n}(-)$ and $\hat{\bar{u}}_{n}(+)$ correspond to an on-shell particle with mass $m_{n}$ and fourmomentum

$$
\hat{p}_{n}^{\mu}=p_{n}^{\mu}-\frac{1}{2} z\left[p_{1}^{b} p_{2}\right]\left\langle p_{n}^{b}+\left|\gamma^{\mu}\right| \eta+\right\rangle .
$$

Momentum conservation is satisfied due to the Schouten identity.

\subsubsection{The remaining helicity configurations}

The shifts for the helicity configurations

$$
\left(1^{-}, 2_{g}^{-}, n^{+}\right), \quad\left(1^{-}, 2_{g}^{+}, n^{+}\right), \quad\left(1^{-}, 2_{g}^{+}, n^{-}\right), \quad\left(1^{-}, 2_{g}^{-}, n^{-}\right)
$$

can be obtained from the helicity configurations

$$
\left(1^{+}, 2_{g}^{+}, n^{-}\right), \quad\left(1^{+}, 2_{g}^{-}, n^{-}\right), \quad\left(1^{+}, 2_{g}^{-}, n^{+}\right), \quad\left(1^{+}, 2_{g}^{+}, n^{+}\right)
$$

by exchanging holomorphic and anti-holomorphic spinors.

\section{$4 \quad$ Large- $z$ behaviour}

We consider $I_{n}(z)$ for $n \geq 4$. $I_{n}(z)$ is a rational function in $z$. We have to show that $I_{n}(z)$ falls off at $z=\infty$ at least with $1 / z$. We will distinguish the cases, where the three particles $1,2_{g}$ and $n$ are 
(i) three gluons,

(ii) two gluons and one quark/anti-quark,

(iii) one gluon and two quarks/anti-quarks, not belonging to the same fermion line or

(iv) one gluon and a quark-anti-quark-pair belonging to the same fermion line.

Let us recall the definition of $I_{n}(z)$ :

$$
I_{n}(z)=\sum_{i=2}^{n-1}\left(\sum_{j=i+1}^{n} 2 \hat{p}_{2} \hat{p}_{j}\right) A_{n}\left(\hat{1}, 3, \ldots, i, \hat{2}_{g}, i+1, \ldots, n-1, \hat{n}\right) .
$$

We note that the factors $\left(2 \hat{p}_{2} \hat{p}_{j}\right)$ are at the worst linear in $z$. A sufficient condition is to show that each amplitude $A_{n}\left(\hat{1}, 3, \ldots, i, \hat{2}_{g}, i+1, \ldots, n-1, \hat{n}\right)$ falls off at $z=\infty$ at least with $1 / z^{2}$. We will show that this holds for the cases (i)-(iii).

However, a $1 / z^{2}$-fall-off behaviour of the amplitudes is not a necessary condition. In fact, in the case (iv) the amplitudes fall off only with $1 / z$. In this case we show through a more sophisticated argument that the full sum $I_{n}(z)$ falls off at $z=\infty$ with $1 / z$.

\subsection{Three gluons}

Let us start with the case $\left(1_{g}, 2_{g}, n_{g}\right)$. The external polarisation vectors contribute a factor $z^{-3}$. The most critical contribution from the vertices and propagators comes from diagrams, where there are only three-gluon vertices along the $z$-flow. For these diagrams there will be along the $z$-flow always one more three-gluon vertex as there are propagators, giving a net factor of $z^{1}$. Therefore we obtain from these diagrams a total contribution of $z^{-3} \cdot z=z^{-2}$. If internally a gluon propagator is replaced by a quark propagator, we have to change at least two three-gluon vertices into quark-gluon vertices. This improves the estimate by a factor $1 / z$. Similarly, the replacement of one three-gluon vertex by a four-gluon vertex results in an improvement in the $z$-behaviour by a factor $1 / z$. We therefore conclude, that the amplitude falls off at $z=\infty$ at least with $1 / z^{2}$.

\subsection{Two gluons and one quark/anti-quark}

The arguments for the cases $\left(1_{q / \bar{q}}, 2_{g}, n_{g}\right)$ and $\left(1_{g}, 2_{g}, n_{q / \bar{q}}\right)$ are very similar to the three gluon case. Although the external polarisations contribute now only a factor $z^{-2}$, the estimate from the vertices and the propagators is now $z^{0}$. Again, the worst diagrams are the ones with a maximal number of three-gluon vertices along the $z$-flow. However, in the case at hand we must have at least one quark-gluon-vertex along the $z$-flow, improving the estimate by a factor $1 / z$. Again we see that the amplitude falls off at $z=\infty$ at least with $1 / z^{2}$.

\subsection{One gluon and two quarks/anti-quarks, not belonging to the same fermi- on line}

Let us now discuss the case $\left(1_{q / \bar{q}}, 2_{g}, n_{q^{\prime} / \bar{q}^{\prime}}\right)$ with one gluon and two quarks/anti-quarks, where the two fermions do not belong to the same fermion line. This sub-case is straightforward: although the external polarisations contribute now only a factor $z^{-1}$, the estimate 
from the vertices and the propagators is now $z^{-1}$. This is due to the fact that we have to change yet another three-gluon vertex into a quark-gluon vertex. Again one concludes that the amplitude falls off at $z=\infty$ at least with $1 / z^{2}$.

\subsection{One gluon and a quark-anti-quark-pair belonging to the same fermion line}

The case $\left(1_{q}, 2_{g}, n_{\bar{q}}\right)$ and $\left(1_{\bar{q}}, 2_{g}, n_{q}\right)$, where the two fermions belong to the same fermion line, are more complicated. Power-counting gives now a factor $z^{-1}$ from the external polarisations and a factor $z^{0}$ from the vertices and propagators. The individual amplitudes fall off as $1 / z$ for large $z$. In this case we show, that the sum $I_{n}(z)$ falls off as $1 / z$ for large $z$. The worst diagrams are the ones, where the $z$-flow of gluon $2_{g}$ goes only through threegluon vertices before it couples to the quark line. We have to show that in the sum the leading $z$-behaviour of these diagrams actually vanishes. For the leading $z$-behaviour we can use an argument of Arkani-Hamed and Kaplan [10]: for large $z$ we may view particles $1,2_{g}$ and $n$ as highly energetic particles moving in a soft background. All vertices along the $z$-flow reduce in this limit to eikonal factors, except the one where the three branches of the $z$-flow meet. In order to see this let us start from particle 2 and consider the first vertex particle 2 meets. This three-gluon vertex couples particle 2 , a current containing only soft particles

$$
J_{\mu}^{\mathrm{soft}}=J_{\mu}^{\mathrm{soft}}(k+1, \ldots, l)
$$

and a current containing the other hard particles 1 and $n$

$$
\hat{J}_{\mu}^{\text {hard }}=\hat{J}_{\mu}^{\text {hard }}(\hat{1}, 3, \ldots, k, l+1, \ldots, \hat{n}) .
$$

In the Feynman rule for the three-gluon vertex we only have to keep the $z$-dependent terms, yielding for the cyclic order $2_{g}, J^{\text {soft }}, \hat{J}^{\text {hard }}$

$$
i\left[-\left(\hat{\varepsilon}_{2} \cdot J^{\mathrm{soft}}\right)\left(\hat{p}_{\mathrm{hard}} \cdot \hat{J}^{\mathrm{hard}}\right)+\left(\hat{p}_{2} \cdot \hat{\varepsilon}_{2}\right)\left(J^{\mathrm{soft}} \cdot \hat{J}^{\mathrm{hard}}\right)-2\left(\hat{\varepsilon}_{2} \cdot \hat{J}^{\mathrm{hard}}\right)\left(\hat{p}_{2} \cdot J^{\mathrm{soft}}\right)\right] .
$$

The contraction of $\hat{p}_{2}$ with $\hat{\varepsilon}_{2}$ vanishes: $\hat{p}_{2} \cdot \hat{\varepsilon}_{2}=0$. Furthermore, the current $\hat{J}^{\text {hard }}$ is conserved and we have $\hat{p}_{\text {hard }} \cdot \hat{J}^{\text {hard }}=0$. This leaves the eikonal contribution

$$
\left(\hat{\varepsilon}_{2} \cdot \hat{J}^{\text {hard,amputated }}\right)\left(-\frac{2 \hat{p}_{2} \cdot J^{\text {soft }}}{\left(\hat{p}_{2}+p_{\text {soft }}\right)^{2}}\right)
$$

with

$$
\hat{J}^{\text {hard,amputated }}=i\left(\hat{p}_{2}+p_{\text {soft }}\right)^{2} \hat{J}^{\text {hard }} .
$$

We may then repeat the argument with the next three-gluon vertex. A similar argument can be given for the $z$-flow along the quark line. Let us start at particle 1 and let us assume that this particle is a quark. We consider the first vertex particle 1 meets. This is a quark-gluon vertex, connecting particle 1, a gluon current containing only soft particles

$$
J_{\mu}^{\mathrm{soft}}=J_{\mu}^{\mathrm{soft}}(3, \ldots, k)
$$


and a hard spinorial current containing the other two hard particles 2 and $n$ :

$$
\hat{V}^{\text {hard }}=\hat{V}^{\text {hard }}(k+1, \ldots, \hat{2}, \ldots, n-1, \hat{n}) .
$$

Let us further define the hard amputated spinorial current as

$$
\hat{V}^{\text {hard }}=i \frac{\left(\not_{1}+\not \not_{\text {soft }}\right)+m}{\left(\not \not_{1}+\not p_{\text {soft }}\right)^{2}-m^{2}} \hat{V}^{\text {hard,amputated }}
$$

Again we may neglect soft momenta in the numerator and we find

$$
\begin{aligned}
& -\hat{\bar{u}}_{1} \gamma^{\mu} \frac{\not \not_{1}+m}{\left(\not \not_{1}+\not p_{\text {soft }}\right)^{2}-m^{2}} \hat{V}^{\text {hard,amputated }} J_{\mu}^{\text {soft }}= \\
& \quad\left(-\frac{2 \hat{p}_{1} \cdot J^{\text {soft }}}{\left(\not p_{1}+\not p_{\text {soft }}\right)^{2}-m^{2}}\right) \hat{\bar{u}}_{1} \hat{V}^{\text {hard,amputated }}+\frac{\hat{\bar{u}}_{1}\left(\not \not_{1}-m\right) \gamma^{\mu} \hat{V}^{\text {hard,amputated }}}{\left(\not p_{1}+\not p_{\text {soft }}\right)^{2}-m^{2}} J_{\mu}^{\text {soft }} .
\end{aligned}
$$

In the first term on the right-hand side we recognise an eikonal factor, the second term vanishes due to the Dirac equation. As before, we may repeat the argument with the next quark-gluon vertex.

The argument for the branch with the external anti-quark at position $n$ is identical and not repeated here. The $\left(1_{\bar{q}}, 2_{g}, n_{q}\right)$-case is very similar and not discussed in detail.

The eikonal factors go to a constant for large $z$ and we are left with a quark-gluon vertex contracted for the $\left(1_{q}, 2_{g}, n_{\bar{q}}\right)$-case with $\hat{\bar{u}}_{1}, \hat{\varepsilon}_{2}$ and $\hat{v}_{n}$. Let us denote this contribution by

$$
O_{3}=i \hat{\bar{u}}_{1} \gamma_{\mu} \hat{v}_{n} \hat{\varepsilon}_{2}^{\mu} \text {. }
$$

The quantity $O_{3}$ falls off like $1 / z$ for large $z$. It is important to note, that $O_{3}$ occurs in every amplitude contributing to $I_{n}(z)$ in the $\left(1_{q}, 2_{g}, n_{\bar{q}}\right)$-case. It may therefore be taken out of the sum, and we have to show that the remaining sum goes to a constant for large $z$. The remaining sum involves only the Lorentz invariants $2 \hat{p}_{2} \hat{p}_{j}$ and the eikonal factors. The proof is given in appendix B.

\section{The proof by induction}

In this section we prove the fundamental BCJ-relation by induction. With the preparations of section 3 and section 4 we can do this independently of the helicity configurations and the masses. This is possible, since we have for $I_{n}(z)$ for all helicity configurations and all masses a $1 / z$-behaviour for large $z$. However, we would like to point out one subtle point for massive quarks: we would like to show that the fundamental BCJ-relations holds for all helicities of the massive quark. The naive way to show this would be to fix a spin quantisation axis through a choice of reference spinors $|q+\rangle$ and $\langle q+|$ and to show the BCJrelation for the helicities "+" and "-" with respect to these reference spinors. This is not what we are doing. The attentive reader of sections 3.4.1-3.4.4 might have noticed, that the "+"- and "-"-helicities refer to different reference spinors. This is o.k., since amplitudes with different spin quantisation axes are related through eq. (3.6) and eq. (3.8). Therefore 
it is sufficient to know two independent amplitudes (say "+"-helicity with respect to $q$ and "-"-helicity with respect to $\tilde{q}$ ) in order to know all amplitudes with spin quantisation axes $q$ and $\tilde{q}$. This remark applies to each external particle individually and covers all possible cases for the external particles 1 and $n$, where we can have out of these two particles either zero, one or two massive particles. In the latter case the masses may be equal or not.

\subsection{Induction start: the case $n=3$}

To start the proof by induction we consider the case $n=3$. Throughout this paper we work with complex external momenta. The external momenta satisfy momentum conservation

$$
p_{1}+p_{2}+p_{3}=0
$$

and the on-shell conditions

$$
p_{1}^{2}=m^{2}, \quad p_{2}^{2}=0, \quad p_{3}^{2}=m^{2} .
$$

Particle $2_{g}$ will always be a gluon and is therefore massless. Particles 1 and 3 may be massless or massive. In the massive case, particles 1 and 3 are necessarily a quark-antiquark pair of the same flavour. Therefore particles 1 and 3 will have the same mass $m$. For $n=3$ external particles the momentum configurations satisfying eq. (5.1) and eq. (5.2) are in general complex. The fundamental BCJ-relation reduces to

$$
2 p_{2} p_{3} A_{3}\left(1,2_{g}, 3\right)=0 \text {. }
$$

For generic external momenta $A_{3}\left(1,2_{g}, 3\right)$ is finite and

$$
2 p_{2} p_{3}=\left(p_{2}+p_{3}\right)^{2}-m^{2}=p_{1}^{2}-m^{2}=0 .
$$

\subsection{The induction step}

We now show that

$$
I_{j}(0)=0
$$

holds for $j=n$, provided it holds for all $j<n$. We start from eq. (2.12)

$$
I_{n}(0)=\frac{1}{2 \pi i} \oint_{z=0} \frac{d z}{z} I_{n}(z),
$$

where the contour is a small counter-clockwise circle around $z=0$. Deforming the contour to a large circle at infinity and the residues at the finite poles $z_{\alpha} \neq 0$ we obtain

$$
I_{n}(0)=B-\sum_{\alpha} \operatorname{res}\left(\frac{I_{n}(z)}{z}\right)_{z_{\alpha}},
$$

where $B$ denotes the contribution from the large circle at infinity. In section 4 we have shown that $I_{n}(z)$ falls off at least with $1 / z$ for $z \rightarrow \infty$ and therefore

$$
B=0 .
$$


It will be convenient to introduce the following notation for the various factorisation channels:

$$
\begin{aligned}
& A_{n}(\hat{1}, 2, \ldots, k, \hat{P} \mid-\hat{P}, k+1, \ldots, n-1, \hat{n})= \\
& \quad \sum_{\lambda} A_{k+1}(\hat{1}, 2, \ldots, k, \hat{P}) \frac{i}{P^{2}} A_{n-k+1}(-\hat{P}, k+1, \ldots, n-1, \hat{n}),
\end{aligned}
$$

together with the convention that the hatted quantities are evaluated at $z=z_{\alpha}$. The sum is over the helicity of the intermediate particle. Let us look at the $z$-momentum flow for a three-particle BCFW-shift. For each diagram we may divide the $z$-dependent propagators into three segments. Each segment starts at the common vertex, where the $z$-dependent momentum flow meets and goes outwards towards the particles $1,2_{g}$ and $n$. We may use these segments to divide the finite residues into three groups and we write

$$
I_{n}(0)=R_{1}+R_{2}+R_{n}
$$

with

$$
\begin{aligned}
R_{1}= & \sum_{i=2}^{n-1}\left(\sum_{j=i+1}^{n} 2 \hat{p}_{2} \hat{p}_{j}\right) \sum_{k=3}^{i} A_{n}\left(\hat{1}, 3, \ldots, k, \hat{P} \mid-\hat{P}, k+1, \ldots, i, \hat{2}_{g}, i+1, \ldots, n-1, \hat{n}\right), \\
R_{2}= & \sum_{i=2}^{n-1}\left(\sum_{j=i+1}^{n} 2 \hat{p}_{2} \hat{p}_{j}\right) \\
& \times \sum_{k=2}^{i} \sum_{\substack{l=i \\
(k, l) \neq(i, i)}}^{n-1} A_{n}\left(k+1, \ldots, i, \hat{2}_{g}, i+1, \ldots, l, \hat{P} \mid-\hat{P}, l+1, \ldots, n-1, \hat{n}, \hat{1}, 3, \ldots, k\right), \\
R_{n}= & \sum_{i=2}^{n-1}\left(\sum_{j=i+1}^{n} 2 \hat{p}_{2} \hat{p}_{j}\right) \sum_{k=i}^{n-2} A_{n}\left(\hat{1}, 3, \ldots, i, \hat{2}_{g}, i+1, \ldots, k, \hat{P} \mid-\hat{P}, k+1, \ldots, n-1, \hat{n}\right) .
\end{aligned}
$$

Let us first look at $R_{1}$. We may exchange the summation over $i$ and $k$ as

$$
\sum_{i=2}^{n-1} \sum_{k=3}^{i} f(i, k)=\sum_{k=3}^{n-1} \sum_{i=k}^{n-1} f(i, k) \text {. }
$$

One obtains

$$
R_{1}=\sum_{k=3}^{n-1} \sum_{i=k}^{n-1}\left(\sum_{j=i+1}^{n} 2 \hat{p}_{2} \hat{p}_{j}\right) A_{n}\left(\hat{1}, 3, \ldots, k, \hat{P} \mid-\hat{P}, k+1, \ldots, i, \hat{2}_{g}, i+1, \ldots, n-1, \hat{n}\right) .
$$

We recognise the fundamental BCJ relation for $(n-k+2)$ external particles. For $k \geq 3$ we have $(n-k+2)<n$. We may therefore use the induction hypothesis and we conclude

$$
R_{1}=0
$$


The argument for $R_{n}$ is very similar. We first exchange the summation indices as

$$
\sum_{i=2}^{n-1} \sum_{k=i}^{n-2} f(i, k)=\sum_{k=2}^{n-2} \sum_{i=2}^{k} f(i, k) \text {. }
$$

We then obtain

$$
\begin{aligned}
R_{n} & =-\sum_{k=2}^{n-2} \sum_{i=2}^{k}\left(\sum_{j=1}^{i} 2 \hat{p}_{2} \hat{p}_{j}\right) A_{n}\left(\hat{1}, 3, \ldots, i, \hat{2}_{g}, i+1, \ldots, k, \hat{P} \mid-\hat{P}, k+1, \ldots, n-1, \hat{n}\right) \\
& =0 .
\end{aligned}
$$

Here we used momentum conservation in the sum over $j$. Again we recognise the fundamental BCJ relation in the form of eq. (2.7). It follows that $R_{n}$ vanishes.

Exchanging the summation indices for $R_{2}$ one obtains

$$
\begin{aligned}
R_{2}= & \sum_{k=2}^{n-2} \sum_{l=k+1}^{n-1} \\
& \times \sum_{i=k}^{l}\left(\sum_{j=i+1}^{n} 2 \hat{p}_{2} \hat{p}_{j}\right) A_{n}\left(k+1, \ldots, i, \hat{2}_{g}, i+1, \ldots, l, \hat{P} \mid-\hat{P}, l+1, \ldots, n-1, \hat{n}, \hat{1}, 3, \ldots, k\right) .
\end{aligned}
$$

We may split the sum over $j$ as

$$
\sum_{j=i+1}^{n} 2 \hat{p}_{2} \hat{p}_{j}=\underbrace{\sum_{j=i+1}^{l} 2 \hat{p}_{2} \hat{p}_{j}}_{A}+\underbrace{\sum_{j=l+1}^{n} 2 \hat{p}_{2} \hat{p}_{j}}_{B}
$$

The terms of type $A$ vanish again by the induction hypothesis

$$
\sum_{i=k}^{l-1}\left(\sum_{j=i+1}^{l} 2 \hat{p}_{2} \hat{p}_{j}\right) A_{l-k+2}\left(\hat{P}, k+1, \ldots, i, \hat{2}_{g}, i+1, \ldots, l\right)=0 .
$$

Note that the sum over $i$ extends only to $(l-1)$, the case $i=l$ contributes only to the terms of type $B$.

For the terms of type $B$ the sum over $j$ is independent of $i$ and may be taken outside the sum over $i$. The sum over $i$ vanishes then due to the $\mathrm{U}(1)$-decoupling relation, given in eq. (2.5):

$$
\left(\sum_{j=l+1}^{n} 2 \hat{p}_{2} \hat{p}_{j}\right) \sum_{i=k}^{l} A_{l-k+2}\left(\hat{P}, k+1, \ldots, i, \hat{2}_{g}, i+1, \ldots, l\right)=0 .
$$

We therefore conclude that

$$
R_{2}=0
$$

Putting the partial results for $R_{1}, R_{2}$ and $R_{n}$ together we find that

$$
I_{n}(0)=0 .
$$

This completes the proof. 


\section{Conclusions}

In this paper we provided a proof of the fundamental BCJ-relation, stated in eq. (2.6), for primitive tree amplitudes in QCD. The proof holds for massless and massive quarks. For the proof we used induction and BCFW-recursion relations.

\section{A Reference spinors for massive particles}

\section{A.1 The helicity configuration $1^{+}, 2_{g}^{+}, n^{-}$}

In this appendix we show that the system

$$
\kappa_{1}\left|q_{1}+\right\rangle=y_{1}\left|p_{n}^{b}+\right\rangle, \quad \kappa_{n}\left\langle q_{n}+\right|=y_{1}\left\langle p_{1}^{b}+\right|+y_{2}\left\langle p_{2}+\right|
$$

has a solution. Expressing $\left\langle p_{2}+\right|$ in terms of $\left\langle l_{1}+\right|$ and $\left\langle l_{n}+\right|$

$$
\left\langle p_{2}+\right|=\frac{\left[p_{2} l_{n}\right]}{\left[l_{1} l_{n}\right]}\left\langle l_{1}+\right|+\frac{\left[l_{1} p_{2}\right]}{\left[l_{1} l_{n}\right]}\left\langle l_{n}+\right|,
$$

we obtain the system of equations

$$
\begin{aligned}
\kappa_{1} & =y_{1} c_{n}, \\
\kappa_{1} \lambda_{1} & =-y_{1} c_{n} \alpha_{1} \lambda_{n}, \\
\kappa_{n} & =y_{1} c_{1}+y_{2} \frac{\left[p_{2} l_{n}\right]}{\left[l_{1} l_{n}\right]}, \\
\kappa_{n} \lambda_{n} & =-y_{1} c_{1} \alpha_{n} \lambda_{1}+y_{2} \frac{\left[l_{1} p_{2}\right]}{\left[l_{1} l_{n}\right]} .
\end{aligned}
$$

The variables $\alpha_{1}$ and $\alpha_{2}$ are defined in eq. (3.14), the variables $c_{1}$ and $c_{n}$ are defined in eq. (3.20). We look for a solution for the variables $\kappa_{1}, \kappa_{n}, y_{1}, y_{2}, \lambda_{1}$ and $\lambda_{n}$. A possible solution is

$$
\begin{aligned}
\kappa_{1} & =\frac{c_{n}}{c_{1}}, & \kappa_{n} & =1+\frac{2 p_{2} l_{n}}{2 l_{1} l_{n}}, \\
y_{1} & =\frac{1}{c_{1}}, & y_{2} & =\frac{\left\langle p_{2} l_{n}\right\rangle}{\left\langle l_{1} l_{n}\right\rangle}, \\
\lambda_{1} & =\frac{p_{n}^{2}\left\langle l_{1}+\left|p_{2}\right| l_{n}+\right\rangle}{\left(2 l_{1} l_{n}\right)^{2}-p_{1}^{2} p_{n}^{2}+\left(2 l_{1} l_{n}\right)\left(2 p_{2} l_{n}\right)}, & \lambda_{n} & =-\frac{2 l_{1} l_{n}\left\langle l_{1}+\left|p_{2}\right| l_{n}+\right\rangle}{\left(2 l_{1} l_{n}\right)^{2}-p_{1}^{2} p_{n}^{2}+\left(2 l_{1} l_{n}\right)\left(2 p_{2} l_{n}\right)} .
\end{aligned}
$$

\section{A.2 The helicity configuration $1^{+}, 2_{g}^{-}, n^{-}$}

In this appendix we show that the system

$$
\kappa_{1}\left|q_{1}+\right\rangle=y_{2}\left|p_{2}+\right\rangle+y_{n}\left|p_{n}^{b}+\right\rangle, \quad \kappa_{n}\left\langle q_{n}+\right|=y_{n}\left\langle p_{1}^{b}+\right|
$$

has a solution. Expressing $\left|p_{2}+\right\rangle$ in terms of $\left|l_{1}+\right\rangle$ and $\left|l_{n}+\right\rangle$

$$
\left|p_{2}+\right\rangle=\frac{\left\langle p_{2} l_{n}\right\rangle}{\left\langle l_{1} l_{n}\right\rangle}\left|l_{1}+\right\rangle+\frac{\left\langle l_{1} p_{2}\right\rangle}{\left\langle l_{1} l_{n}\right\rangle}\left|l_{n}+\right\rangle,
$$


we obtain the system of equations

$$
\begin{aligned}
\kappa_{n} & =y_{n} c_{1}, \\
\kappa_{n} \lambda_{n} & =-y_{n} c_{1} \alpha_{n} \lambda_{1}, \\
\kappa_{1} & =y_{n} c_{n}+y_{2} \frac{\left\langle l_{1} p_{2}\right\rangle}{\left\langle l_{1} l_{n}\right\rangle}, \\
\kappa_{1} \lambda_{1} & =-y_{n} c_{n} \alpha_{1} \lambda_{n}+y_{2} \frac{\left\langle p_{2} l_{n}\right\rangle}{\left\langle l_{1} l_{n}\right\rangle} .
\end{aligned}
$$

A possible solution is

$$
\begin{array}{rlrl}
\kappa_{1} & =1+\frac{2 l_{1} p_{2}}{2 l_{1} l_{n}}, & \kappa_{n}=\frac{c_{1}}{c_{n}} \\
y_{2}=\frac{\left[l_{1} p_{2}\right]}{\left[l_{1} l_{n}\right]}, & y_{n}=\frac{1}{c_{n}}, \\
\lambda_{1}=-\frac{2 l_{1} l_{n}\left\langle l_{1}+\left|p_{2}\right| l_{n}+\right\rangle}{\left(2 l_{1} l_{n}\right)^{2}-p_{1}^{2} p_{n}^{2}+\left(2 l_{1} l_{n}\right)\left(2 l_{1} p_{2}\right)}, & \lambda_{n}=\frac{p_{1}^{2}\left\langle l_{1}+\left|p_{2}\right| l_{n}+\right\rangle}{\left(2 l_{1} l_{n}\right)^{2}-p_{1}^{2} p_{n}^{2}+\left(2 l_{1} l_{n}\right)\left(2 l_{1} p_{2}\right)} .
\end{array}
$$

\section{B The large $z$-behaviour in the eikonal approximation}

Let us consider a theory with massless or massive scalar "hard" particles, denoted by a hat and QCD-like "soft" particles (gluons, quarks, anti-quarks), denoted without a hat. The momenta of the hard particles are of order $z^{1}$, the momenta of the soft particles are of order $z^{0}$. The Feynman rules for this toy theory are as follows: the hard particles interact only through three-valent vertices. The Feynman rule for the three-valent vertex involving three hard particles with the cyclic order $(\hat{1}, \hat{2}, \hat{3})$ is simply $i$, for the cyclic order $(\hat{1}, \hat{3}, \hat{2})$ we have $(-i)$. Furthermore there is a three-valent vertex, involving two hard particles and one soft gluon. The Feynman rule for the cyclic order $(\hat{1}, 2, \hat{3})$ reads

$$
i\left(\hat{p}_{1}^{\mu}-\hat{p}_{3}^{\mu}\right)
$$

There are no vertices involving only one hard particle. The Feynman rules for the vertices involving only soft particles are the standard (cyclic-ordered) QCD Feynman rules, listed in appendix $\mathrm{C}$.

Let us consider the situation of three hard particles $\hat{1}, \hat{2}$ and $\hat{n}$ and $(n-3)$ soft particles $3, \ldots,(n-1)$. We assume particle $\hat{2}$ to be massless and particles $\hat{1}$ and $\hat{n}$ to have the same mass $m$ (which may be zero). We will denote an amplitude in this toy theory by

$$
A_{n}^{\text {eikonal }}(\hat{1}, 3, \ldots, i, \hat{2}, i+1, \ldots, n-1, \hat{n}) \text {, }
$$

and we define

$$
I_{n}^{\text {eikonal }}(z)=\sum_{i=2}^{n-1}\left(2 \hat{p}_{2} \hat{p}_{n}+\sum_{j=i+1}^{n-1} 2 \hat{p}_{2} p_{j}\right) A_{n}^{\text {eikonal }}(\hat{1}, 3, \ldots, i, \hat{2}, i+1, \ldots, n-1, \hat{n}) .
$$


We would like to show that $I_{n}^{\text {eikonal }}(z)$ goes to a constant for large $z$. Then the quantity

$$
\mathrm{O}_{3} \mathrm{I}_{n}^{\mathrm{eikonal}}(z)
$$

with $O_{3}$ defined as in eq. (4.11) falls off like $1 / z$.

It will be convenient to introduce soft currents

$$
J_{\text {soft }}^{\mu}(a, \ldots, b),
$$

involving $(b-a+1)$ soft on-shell particles $a, a+1, \ldots, b$ and one soft off-shell gluon leg. The momentum of this soft current is

$$
P=\sum_{k=a}^{b} p_{k} .
$$

We may group the Feynman diagrams contributing to $I_{n}^{\text {eikonal }}(z)$ into sets, where exactly $r$ soft currents couple to the hard particles $\hat{1}, \hat{2}$ and $\hat{n}$ with $1 \leq r \leq n-3$. Therefore we have a decomposition

$$
I_{n}^{\text {eikonal }}(z)=\sum_{r=1}^{n-3} I_{n, r}^{\text {eikonal }}(z) .
$$

We will show that each contribution $I_{n, r}^{\text {eikonal }}(z)$ individually goes to a constant for large $z$.

Let us discuss $I_{n, r}^{\text {eikonal }}(z)$ with $r$ soft currents $J_{1}^{\text {soft }}, \ldots, J_{r}^{\text {soft }}$ and associated momenta $P_{1}, \ldots, P_{r}$. The cyclic order among the soft currents is respected in each diagram contributing to $I_{n, r}^{\text {eikonal }}(z)$. We will use the notation

$$
P_{a, a+1, \ldots, b}=\sum_{k=a}^{b} P_{k} .
$$

Let us first discuss the situation, where two or more soft currents couple to the hard line $\hat{2}$. These contributions add up to zero in $I_{n, r}^{\text {eikonal }}(z)$. In order to see this, consider the situation, where the two outermost soft currents coupling to $\hat{2}$ are $J_{a}^{\text {soft }}$ and $J_{a+1}^{\text {soft }}$. There are four possibilites, how these soft currents may couple to $\hat{2}$, shown in figure 1 . With the inclusion of the prefactors of the fundamental BCJ-relation, these contributions add up to zero.

Let us now consider the case, where one soft current $J_{a}^{\text {soft }}$ couples to the hard particle $\hat{2}$. Here we get the contribution

$$
-\left(2 \hat{p}_{2} P_{a}\right) \frac{2 \hat{p}_{2} J_{a}^{\mathrm{soft}}}{\left(\hat{p}_{2}+P_{a}\right)^{2}}=2 \hat{p}_{1} J_{a}^{\mathrm{soft}}+2 \hat{p}_{n} J_{a}^{\mathrm{soft}}+\mathcal{O}\left(z^{0}\right) .
$$

We may now add up all contributions and obtain

$$
\begin{aligned}
I_{n, r}^{\text {eikonal }}(z)= & \sum_{i=0}^{r}\left(2 \hat{p}_{2} \hat{p}_{n}+\sum_{j=i+1}^{r} 2 \hat{p}_{2} P_{j}\right)(-1)^{i}\left(\prod_{k=1}^{i} \frac{2 \hat{p}_{1} J_{k}^{\text {soft }}}{2 \hat{p}_{1} P_{1, \ldots, k}}\right)\left(\prod_{l=i+1}^{r} \frac{2 \hat{p}_{n} J_{l}^{\text {soft }}}{2 \hat{p}_{n} P_{l, \ldots, r}}\right) \\
& +\sum_{i=1}^{r}(-1)^{i-1}\left(\prod_{k=1}^{i-1} \frac{2 \hat{p}_{1} J_{k}^{\text {soft }}}{2 \hat{p}_{1} P_{1, \ldots, k}}\right)\left(\prod_{l=i+1}^{r} \frac{2 \hat{p}_{n} J_{l}^{\text {soft }}}{2 \hat{p}_{n} P_{l, \ldots, r}}\right)\left(2 \hat{p}_{1} J_{i}^{\text {soft }}+2 \hat{p}_{n} J_{i}^{\text {soft }}\right) \\
& +\mathcal{O}\left(z^{0}\right) .
\end{aligned}
$$



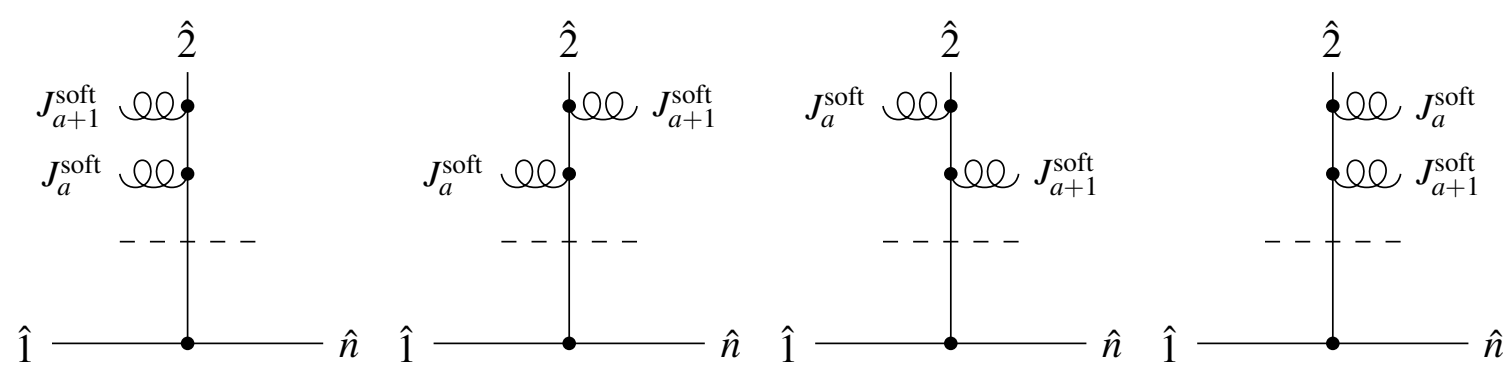

Figure 1. Diagrams showing how the two outermost soft currents $J_{a}^{\text {soft }}$ and $J_{a+1}^{\text {soft }}$ may couple to the hard particle $\hat{2}$. There may be further soft currents attached to the hard lines below the dashed line.

The terms in the first sum come from diagrams, where all soft currents couple either to the hard particle $\hat{1}$ or $\hat{n}$, the terms of the second sum correspond to diagrams, where exactly one soft current couples to the hard particle $\hat{2}$. Noting that

$$
2 \hat{p}_{2} \hat{p}_{n}+\sum_{j=i+1}^{r} 2 \hat{p}_{2} P_{j}=2 \hat{p}_{1} P_{1, \ldots, i}-2 \hat{p}_{n} P_{i+1, \ldots, r}+\mathcal{O}\left(z^{0}\right)
$$

one sees that

$$
I_{n, r}^{\text {eikonal }}(z)=\mathcal{O}\left(z^{0}\right)
$$

as claimed.

\section{Cyclic-ordered Feynman rules}

In this appendix we give a list of the cyclic-ordered Feynman rules. They are obtained from the standard Feynman rules by extracting from each formula the coupling constant and the colour part. The propagators for quark and gluon particles are given by

$$
\begin{aligned}
& \longleftarrow=i \frac{\not p+m}{p^{2}-m^{2}}, \\
& \text { eeeee }=\frac{-i g^{\mu \nu}}{p^{2}} .
\end{aligned}
$$

The cyclic-ordered Feynman rules for the three-gluon and the four-gluon vertices are

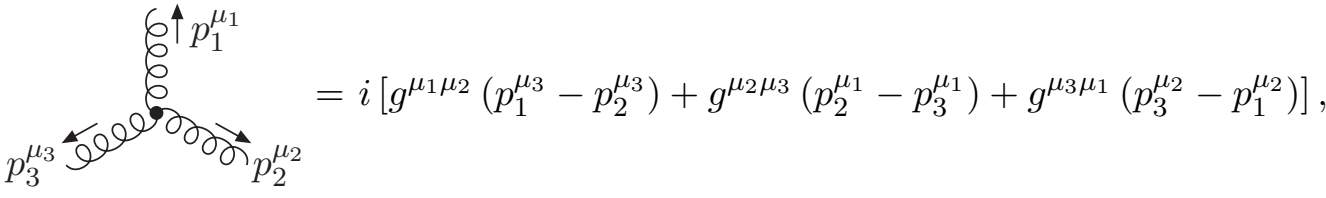

$$
\begin{aligned}
& { }_{\mu_{3}}^{\mu_{4}} \operatorname{6\sigma }_{\sigma^{6}}^{\sigma^{\mu_{1}}}=i\left[2 g^{\mu_{1} \mu_{3}} g^{\mu_{2} \mu_{4}}-g^{\mu_{1} \mu_{2}} g^{\mu_{3} \mu_{4}}-g^{\mu_{1} \mu_{4}} g^{\mu_{2} \mu_{3}}\right] \text {. }
\end{aligned}
$$


The Feynman rule for the quark-gluon vertex is given by

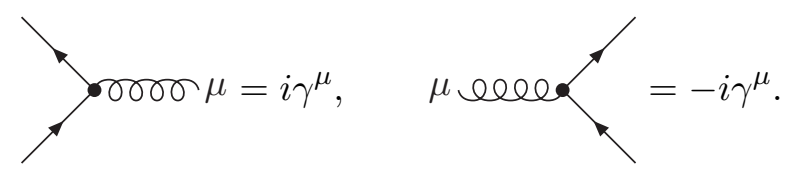

Open Access. This article is distributed under the terms of the Creative Commons Attribution License (CC-BY 4.0), which permits any use, distribution and reproduction in any medium, provided the original author(s) and source are credited.

\section{References}

[1] Z. Bern, L.J. Dixon and D.A. Kosower, One loop corrections to two quark three gluon amplitudes, Nucl. Phys. B 437 (1995) 259 [hep-ph/9409393] [INSPIRE].

[2] C. Reuschle and S. Weinzierl, Decomposition of one-loop QCD amplitudes into primitive amplitudes based on shuffle relations, Phys. Rev. D 88 (2013) 105020 [arXiv:1310.0413] [INSPIRE].

[3] R. Kleiss and H. Kuijf, Multi-Gluon Cross-sections and Five Jet Production at Hadron Colliders, Nucl. Phys. B 312 (1989) 616 [inSPIRE].

[4] Z. Bern, J.J.M. Carrasco and H. Johansson, New Relations for Gauge-Theory Amplitudes, Phys. Rev. D 78 (2008) 085011 [arXiv:0805.3993] [InSPIRE].

[5] B. Feng, R. Huang and Y. Jia, Gauge Amplitude Identities by On-shell Recursion Relation in S-matrix Program, Phys. Lett. B 695 (2011) 350 [arXiv: 1004.3417] [INSPIRE].

[6] N.E.J. Bjerrum-Bohr, P.H. Damgaard and P. Vanhove, Minimal Basis for Gauge Theory Amplitudes, Phys. Rev. Lett. 103 (2009) 161602 [arXiv:0907.1425] [INSPIRE].

[7] S. Stieberger, Open \& Closed vs. Pure Open String Disk Amplitudes, arXiv:0907.2211 [INSPIRE].

[8] Y. Jia, R. Huang and C.-Y. Liu, U(1)-decoupling, KK and BCJ relations in $\mathcal{N}=4 S Y M$, Phys. Rev. D 82 (2010) 065001 [arXiv: 1005.1821] [INSPIRE].

[9] Y.-X. Chen, Y.-J. Du and B. Feng, A Proof of the Explicit Minimal-basis Expansion of Tree Amplitudes in Gauge Field Theory, JHEP 02 (2011) 112 [arXiv:1101.0009] [INSPIRE].

[10] N. Arkani-Hamed and J. Kaplan, On Tree Amplitudes in Gauge Theory and Gravity, JHEP 04 (2008) 076 [arXiv: 0801.2385] [INSPIRE].

[11] N. Arkani-Hamed, F. Cachazo and J. Kaplan, What is the Simplest Quantum Field Theory?, JHEP 09 (2010) 016 [arXiv:0808.1446] [INSPIRE].

[12] C. Cheung, On-Shell Recursion Relations for Generic Theories, JHEP 03 (2010) 098 [arXiv:0808.0504] [INSPIRE].

[13] S.G. Naculich, Scattering equations and BCJ relations for gauge and gravitational amplitudes with massive scalar particles, JHEP 09 (2014) 029 [arXiv:1407.7836] [INSPIRE].

[14] H. Johansson and A. Ochirov, Color-Kinematics Duality for QCD Amplitudes, arXiv: 1507.00332 [INSPIRE].

[15] P. Mastrolia, A. Primo, U. Schubert and W.J.T. Bobadilla, Off-shell Color-Kinematics Duality, arXiv:1507.07532 [INSPIRE]. 
[16] M.L. Mangano and S.J. Parke, Multiparton amplitudes in gauge theories, Phys. Rept. 200 (1991) 301 [hep-th/0509223] [INSPIRE].

[17] T. Schuster, Color ordering in QCD, Phys. Rev. D 89 (2014) 105022 [arXiv:1311.6296] [INSPIRE].

[18] L.J. Dixon, J.M. Henn, J. Plefka and T. Schuster, All tree-level amplitudes in massless QCD, JHEP 01 (2011) 035 [arXiv: 1010.3991] [INSPIRE].

[19] T. Melia, Dyck words and multiquark primitive amplitudes, Phys. Rev. D 88 (2013) 014020 [arXiv: 1304.7809] [INSPIRE].

[20] T. Melia, Getting more flavor out of one-flavor QCD, Phys. Rev. D 89 (2014) 074012 [arXiv: 1312.0599] [INSPIRE].

[21] R. Britto, F. Cachazo, B. Feng and E. Witten, Direct proof of tree-level recursion relation in Yang-Mills theory, Phys. Rev. Lett. 94 (2005) 181602 [hep-th/0501052] [INSPIRE].

[22] K. Risager, A Direct proof of the CSW rules, JHEP 12 (2005) 003 [hep-th/0508206] [INSPIRE].

[23] C. Schwinn and S. Weinzierl, On-shell recursion relations for all Born QCD amplitudes, JHEP 04 (2007) 072 [hep-ph/0703021] [INSPIRE].

[24] F. del Aguila and R. Pittau, Recursive numerical calculus of one-loop tensor integrals, JHEP 07 (2004) 017 [hep-ph/0404120] [INSPIRE].

[25] A. van Hameren, J. Vollinga and S. Weinzierl, Automated computation of one-loop integrals in massless theories, Eur. Phys. J. C 41 (2005) 361 [hep-ph/0502165] [InSPIRE]. 